\title{
Clinical Outcome of Benign Cardiac Tumors in Infants During A 13 Years' Experience: Impact of Prenatal Diagnosis
}

\author{
Camilla Sandrini ${ }^{1}$, Stiljan Hoxha ${ }^{2}$, Lucia Rossetti ${ }^{1}$, Mara Pilati ${ }^{1}$, Maria Antonia Prioli ${ }^{1}$, Flavio Luciano Ribichini ${ }^{1}$, \\ Giuseppe Faggian² and Giovanni Battista Luciani*2 \\ ${ }^{1}$ Department of Medicine, University of Verona, Italy \\ ${ }^{2}$ Department of Surgery, Pediatrics and Gynecology University of Verona, Italy
}

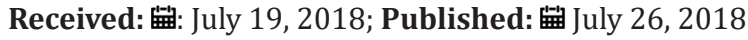

*Corresponding author: Giovanni Battista Luciani, Division of Cardiac Surgery, Department of Surgery, University of Verona, Italy

\begin{abstract}
Background: Primary cardiac tumors (PCT) in children are rare disorders. Prenatal diagnosis is feasible. Most of them have favorable prognosis, but some still prove fatal.Objective: To define clinical outcome of benign PCT and the possible impact of prenatal diagnosis.

Methods: 14 cases were collected between December 2005 and August 201. We included patients with pre and postnatal diagnosis, medically and surgically managed. Pre and postnatal examinations, surgical procedures, clinical information before and after surgery, histological diagnoses were reviewed.
\end{abstract}

Results: Seventy-two percent of PCT were detected prenatally. Median follow-up time was 3,13 years. In the medical managed group, one had complete spontaneous regression, 3 had spontaneous reduction, 2 underwent efficacious Enviroximes treatment and one was lost to follow-up. 46\% of cases needed surgery. In two infants, perioperative support with V-A ECMO and iNO was necessary. Complete excision of the mass was possible in 5 cases. Rate of hospital death was 20\%. All but one late survivors were symptom-free at last clinical assessment. Clinical relevant recurrence of mixoma occurred in one case.

Conclusion: Benign PCT have extremely diverse course, some proving fatal in spite of benign histology. Prenatal diagnosis is crucial to plan delivery and neonatal management, as some benign PCT require intensive care. Complete excision of the mass usually is feasible but may be limited by young age and extent of cardiac infiltration. Even if patients are generally well after surgery, long term follow up is mandatory as some masses recur

Keywords: Congenital Heart Disease; Primary Cardiac Tumors; Prenatal Diagnosis; Clinical Outcome

Abbreviations: PCT: Primary Cardiac Tumors; CHD: Congenital Heart Disease; FU: Follow Up; CT: Computed Tomography; CMRI: Cardiac Magnetic Resonance Imaging; NYHA: New York Heart Association; TOP: Termination of Pregnancy; SD: Standard Deviation; ECG: Electrocardiography; PGE: Prostaglandin; Ino: Inhaled Nitric Oxide; ASD: Atrial Septal Defect; VSD: Ventricular Septal Defect; V-A ECMO: Veno-Arterial Extracorporeal Membrane Oxygenation; CAT: Calcified Amorphous Tumor

\section{Introduction}

Primary cardiac tumors (PCT) in children are an extremely rare and potentially life-threatening disease with an incidence ranging from $0.027 \%$ to $0.08 \%[1,2]$. Cardiac tumors have bizarre clinical behavior and may be asymptomatic even when of large dimensions, thus the clinical course can vary ranging from spontaneous regression to fatal [1-4]. Urgent surgical excision of the mass and extracorporeal life support is sometimes needed but complete excision is not always possible especially in some anatomical patterns [4]. Prenatal diagnosis of PCT is possible, even if not always feasible, often due to familiar history of fetal tumors, suspicion of congenital heart disease (CHD), fetal heart failure or fetal arrhythmias [5]. Although differential diagnosis between different types of PCT is nowadays possible through noninvasive image techniques, the gold standard remains the histological confirmation [6-9]. The aim of the current study was to define clinical outcome of benign PCT diagnosed at our Institution and the possible impact of prenatal diagnosis.

\section{Material}

Institutional Review Board approval was obtained for the conduct of this study and the board waived the need for patient consent. We retrospectively searched for all cases of PCT observed at our Institution between January 2005 and August 2018 in patients aged less than 18 years. Information included demographic data, age at diagnosis, clinical findings, imaging findings, surgical details, drug use, postoperative outcome, histological diagnosis, when possible, and follow up (F/U) examination. Complete cardiac 
echocardiography was the main method used for diagnosis. All echocardiographic examinations performed during the study period were reviewed. Segmental study of the cardiac anatomy was used in both pre and postnatal echography. Prenatal echocardiographic diagnostic data consisted in definition of the mass (isolated/ multiple, dimension, site, echogenic characteristic, shape, margins, vascularization, relationship with intra-cardiac structure), definition of presence or absence of associated CHD (2D and Doppler interrogation of vascular structures), study of fetal cardiac rhythm (Doppler analysis of cardiac inflow and outflow, M-mode study of atrial and ventricular activation), study of hemodynamic impact of the mass (presence or cardiomegaly, inflow or outflow obstruction, valves dysfunction, systolic or diastolic ventricular dysfunction, pericardial effusion, abnormalities in the flow of hepatic and sovra-hepatic veins and venous duct).

For each examination, study of the situs visceralis, four chamber view, left and right outflow tracts, short axis view of the ventricle and of great vessels, bicaval section, aortic arch and ductal arch definition, hepatic, sovra-hepatic and venous duct flow were collected. If necessary, obstetric ultrasound echography was performed to better define fetal hydrops, extra-cardiac associated malformations and fetal growth. The same data were collected in the postnatal period by using subcostal, parasternal long and short axis, apical and supraclavicular view by 2D, M-mode, Doppler, color-Doppler and tissue-Doppler technology. Second line imaging investigation by cardiac computed tomography (CT) was performed in 2 pts with suspected tumor infiltration of surrounding structures in order to define location, morphology and boundaries of the tumor. Cardiac magnetic resonance imaging (cMRI) was performed in two patients. Following diagnosis, patients were medically or surgically managed based on clinical behavior and instrumental data. F/U was offered to all patients. Evaluation included physical examinations (NYHA functional class), electrocardiographic features, echocardiography data, pharmacological treatment and physical activity. Fourteen patients met our inclusion criteria during the period of time covered by the study. 8 cases were male, 5 cases were female, one decided for termination of pregnancy (TOP). Demographic data are summarized in (Table 1).

\section{Statistical Analysis}

Descriptive analysis was used. Continuous variables were expressed as mean \pm SD. Categorical variables were reported as absolute numbers and percentage.

\section{Results}

\section{Prenatal Diagnosis}

Ten PCTs $(10 / 14,72 \%)$ were detected prenatally with a mean gestational age at diagnosis of 24 weeks of amenorrhea (18-29 weeks). Referral indications for fetal echocardiography were suspicion of CHD at second trimester obstetric ultrasound screening and familiar history of CHD. In one case, fetal echocardiography performed for high risk of chromosomal abnormalities was deemed normal but PCT was diagnosed postnatally. 6 cases of PCTs detected prenatally had associated comorbidities: 4 had tuberous sclerosis, one had renal agenesis and one had skeletal malformations of hands and feet associated to microdeletion of chromosome 22. At postnatal examination, others associated cardiac anomalies were depicted: atrial septal defect in two patients, ventricular septal defect and atrial septal defect in one other patient. No one of our patients diagnosed prenatally had fetal arrhythmias. Only one case developed in utero pericardial effusion, mild to moderate tricuspid valve insufficiency, moderate left ventricular dysfunction and ascites. All pregnancies were successfully delivered with a mean gestational age of 39 weeks of amenorrhea ( $\max 40$ weeks, min 36 weeks), except one young women who decided for termination of pregnancy following the diagnosis of a large PCT in the left ventricle at 20 weeks of amenorrhea. All out of one pregnancies were normally delivered. One case needed caesarian section because of fetal hemodynamic instability.

\section{Postnatal Diagnosis}

Four cases $(4 / 13,31 \%)$ were detected after birth. Mean age at postnatal diagnosis was $1.5 \pm 0.86$ yrs. (range, 10 days -5.5 years). Screening for cardiac malformation in the context of extra-cardiac abnormalities was the indication for echocardiography in two asymptomatic cases detected in neonatal period. The remaining two diagnoses were made in older age because of signs or symptoms (exercise induced dyspnea and systolic murmur).

\section{Clinical Findings}

Symptoms were present in 5 patients $(5 / 13,38 \%)$, comprising mild exercise induced dyspnea in one postnatal detected child $(1 / 5$, $20 \%$ ), progressive hemodynamic instability due to severe tricuspid valve obstruction $(2 / 5,40 \%)$, progressive hemodynamic instability due to right ventricular outflow tract obstruction $(1 / 5,20 \%)$ and cyanosis due to interatrial right to left shunt due to tricuspid valve obstruction $(1 / 5,20 \%)$ in the 4 neonatal cases. All symptomatic neonates had prenatal diagnosis of PCT. EKG revealed biventricular hypertrophy in one case. Physical examination revealed only a mild systolic murmur in two cases. The older child with symptoms did not require pharmacological or mechanical circulatory support. On the contrary, neonates needed cardio-circulatory and respiratory support with mechanical ventilation $(3 / 4,75 \%)$, prostaglandin E (PGE) infusion $(3 / 4,75 \%)$, inhaled nitric oxide (iNO) use $(2 / 4$, $50 \%)$ and balloon atrial septostomy $(1 / 4,25 \%)$, Everolimus therapy $(1 / 4,25 \%)$. Urgent surgical strategy was undertaken in one infant for impending embolization of the large mass. Emergency surgical strategy was required in the other three neonates due to severe hemodynamic impairment: 2 newborn, one with the unresectable mass, died because of cardiogenic shock, in one case, and multiorgan failure and septic shock, in the other case.

Eight patients $(8 / 13,62 \%)$ were symptoms-free at the time of diagnosis. Five of the symptom-free survivors had received prenatal diagnosis, while the remaining three had postnatal diagnosis due to the presence of extra-cardiac malformations, 2 cases, and of systolic murmur, 1 case. Peripheral arterial pressure, heart rate, oxygen blood delivery and ECG were normal in all asymptomatic patients. Drug therapy with Everolimus was deemed necessary only in two neonates with voluminous non-obstructive rhabdomyomas. Surgery was performed on elective criteria in 2 patients (one due to extreme mobility of a small mass in the left atrial appendage and the second one due to concomitant repair of outlet ventricular 
septal defect). Cardiac tumors were found as multiple masses in 8 patients and as single mass in 6 patients. Right heart was primarily involved in 5 patients, left heart in 5 patients, while 4 pts had biventricular involvement. The masses were localized mostly in the ventricles $(12 / 14,86 \%)$ with only 2 cases localized only in the atria $(2 / 14,14 \%)$ (Figures 1-3). No one had extra-cardiac location. Associated cardiac anomalies were present in 4 patients (31\%) (Table 1). One case had familial recurrence of PCT. One case had a recurrence of clinical relevant mixoma (Figures 4-6). Type of PCT are summarized in (Table 2).

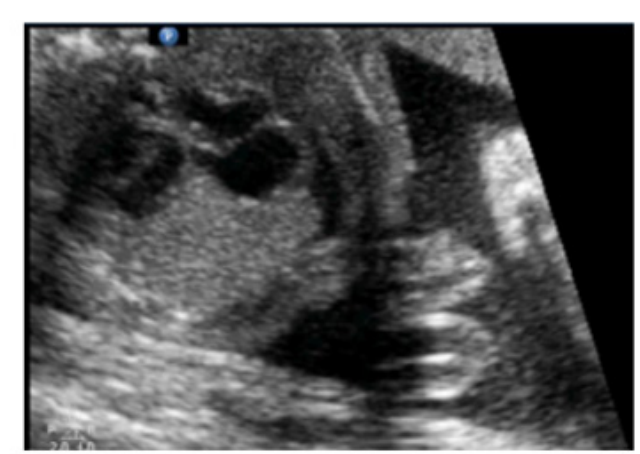

A

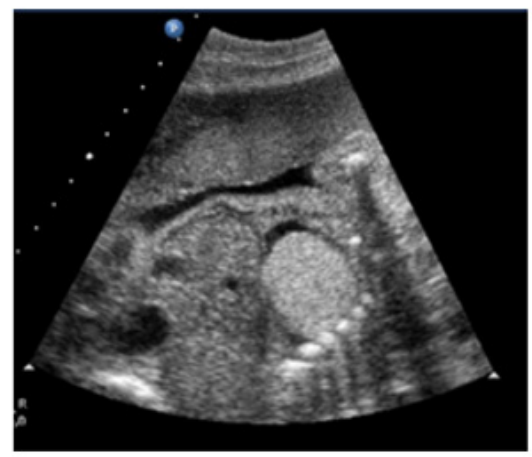

B

Figure 1:

A. Biventricular Rhabdomyoma.

B. Fetal Echocardiography.

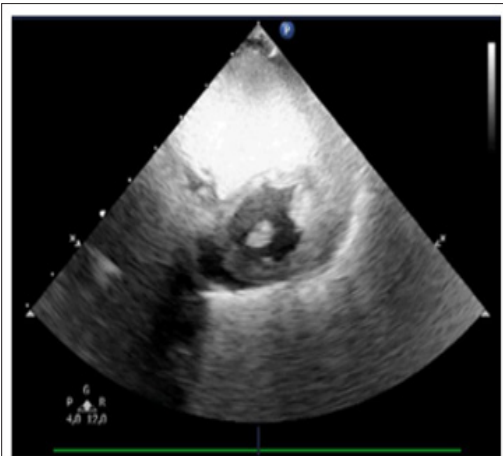

A

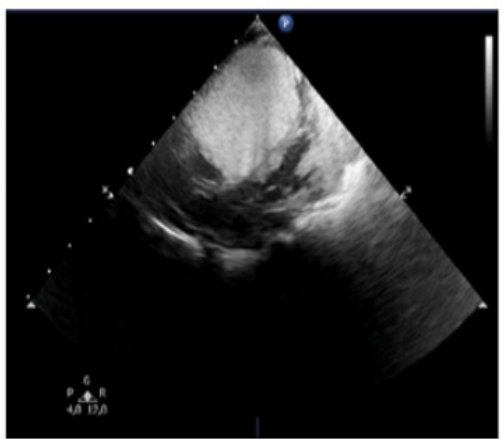

B

Figure 2:

A. Biventricular Rhabdomyoma.

B. Postnatal Echocardiography.

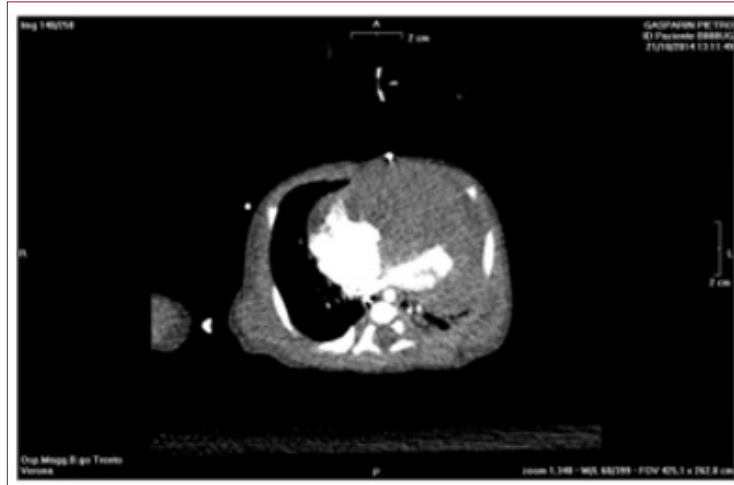

A

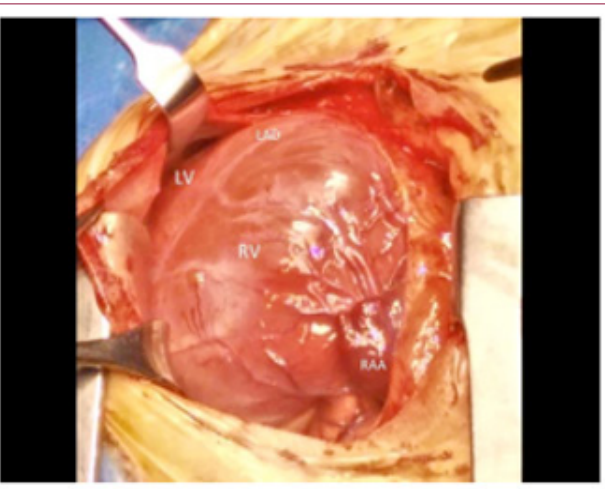

B

Figure 3: Biventricular Rhabdomyoma Showing Diffuse and Unresectable Biventricular Infiltration.

A. Preoperative CT Scan.

B. Intraoperative View. 


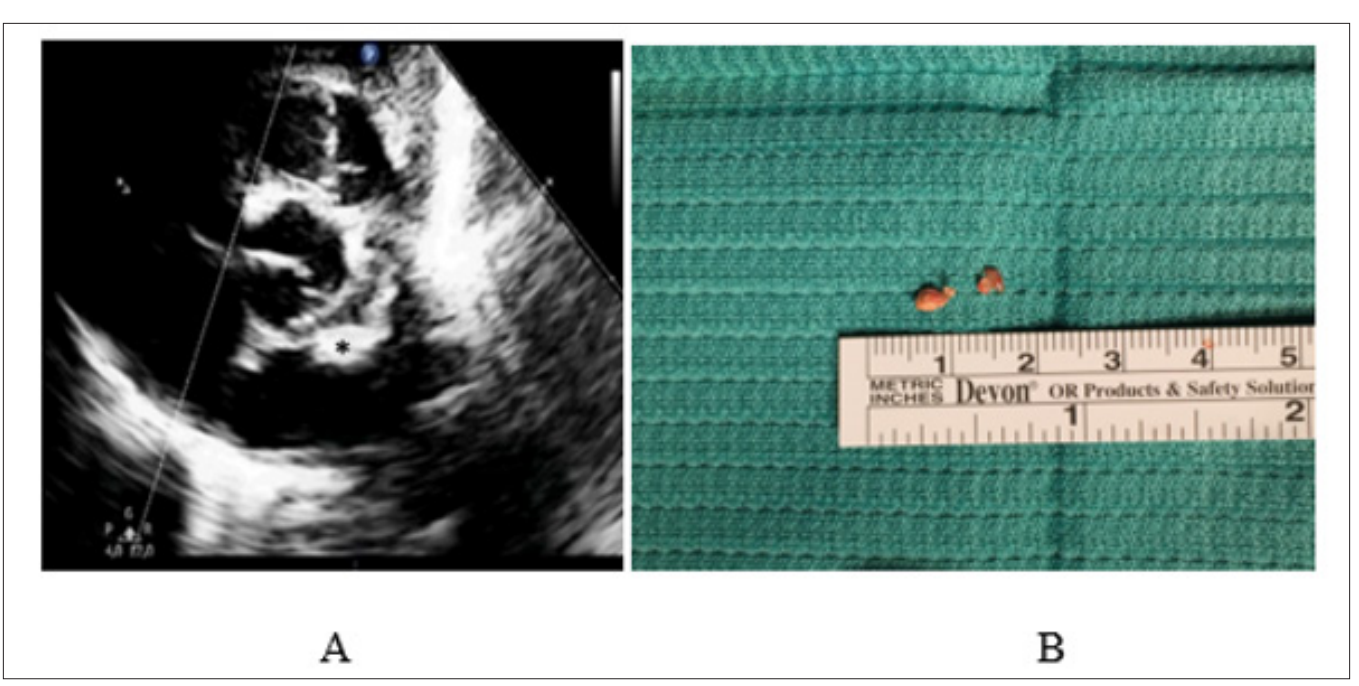

Figure 4: Calcified Amorphous Tumor of The Heart (CAT).

A. Preoperative Two-Dimensional Echocardiogram Showing A Club-Shaped (Asterisk) Left Atrial Mass, With Very Thin Pedicle and Extremely Mobile Appearance.

B. Intra-Operative View of The Gross Anatomic Aspect of The Mass and Pedicle, Subsequently Diagnosed as CAT at Histological Examination.

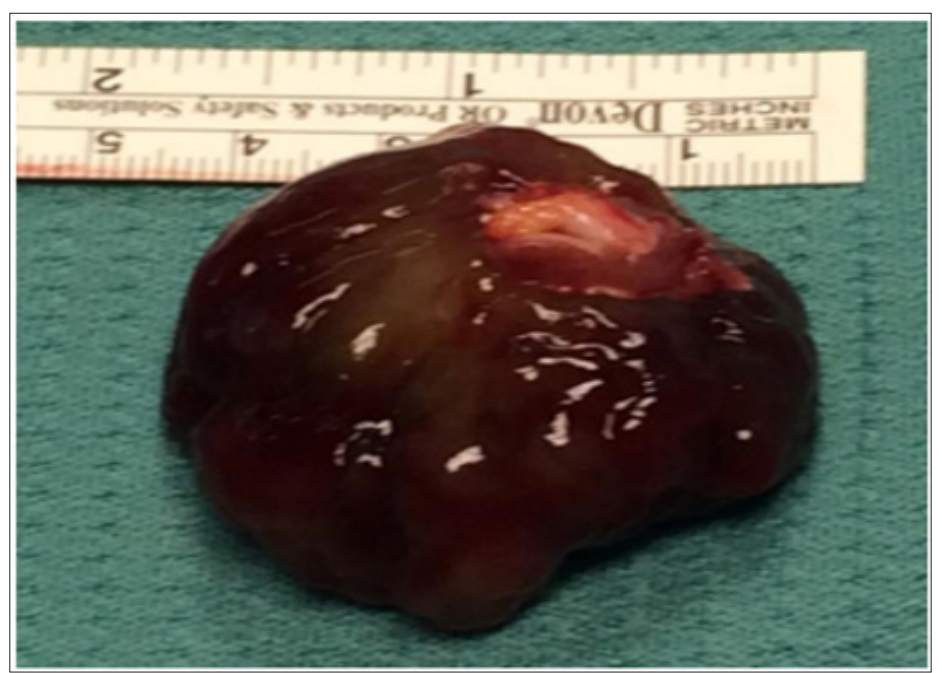

Figure 5: Giant Left Atrial Myxoma, Gross Anatomic Aspect.

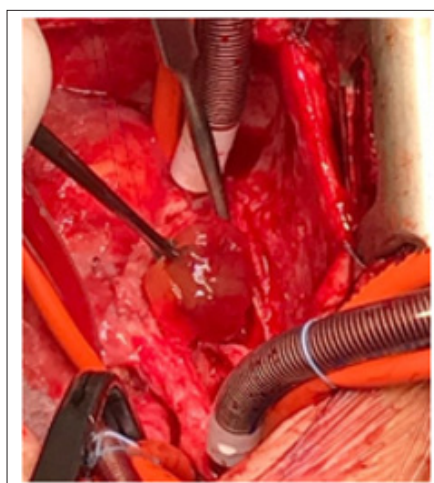

A

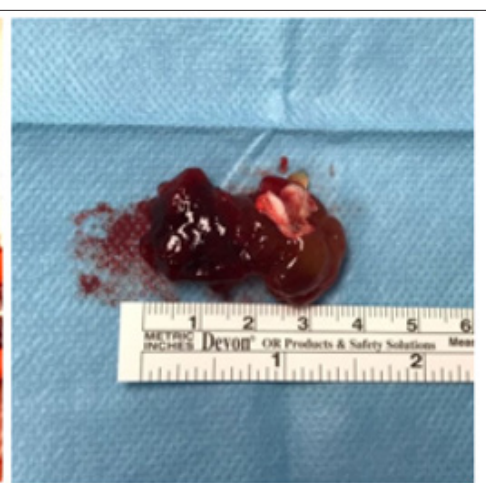

B

Figure 6: Recurrence of Mixoma.

A. Intra-Operative Vision.

B. Macroscopic Evaluation. 
Table 1: Demographic Data.

\begin{tabular}{|c|c|}
\hline Variable & Mean \pm SD; OR N (\%) \\
\hline Number of cases with PCT & 14 \\
\hline Termination of pregnancy & $1(7 \%)$ \\
Prenatal diagnosis & $8 / 5(1$ not know $)$ \\
Mean age at prenatal diagnosis & $10(72 \%)$ \\
\hline Mean age at postnatal diagnosis & 24 wks \\
\hline Symptoms at diagnosis & $1.5 \pm 0.86 \mathrm{yrs}$ \\
Associated cardiac anomalies & $5(38 \%)$ \\
ASD & $3(23 \%)$ \\
ASD+VSD & 2 \\
Extra-cardiac anomalies & 1 \\
Tuberous sclerosis & $6(43 \%)$ \\
Renal agenesis & 4 \\
Skeletal malformations & 1 \\
Surgically treated & 1 \\
Mean age at surgery & $6(46 \%)$ \\
\hline
\end{tabular}

Table 2: Histological Data

\begin{tabular}{|c|c|}
\hline Case & Histological Data \\
\hline 1 & rhabdomyoma (only suspected) \\
\hline 2 & rhabdomyoma (only suspected) \\
\hline 3 & not known (TOP) \\
\hline 4 & rhabdomyoma \\
\hline 5 & CAT \\
\hline 6 & fetal type rhabdomyoma \\
\hline 7 & fetal type rhabdomyoma \\
\hline 8 & rhabdomyoma (only suspected) \\
\hline 9 & rhabdomyoma (only suspected) \\
\hline 10 & rhabdomyoma (only suspected) \\
\hline 11 & rhabdomyoma (only suspected) \\
\hline 12 & rhabdomyoma (only suspected) \\
\hline 13 & \\
\hline 14 & \\
\hline
\end{tabular}

\section{Surgical Management}

Surgical intervention was necessary in six patients $(6 / 13$, $46 \%$ ), because of obstruction associated with hemodynamic instability or impending embolization and it was performed on an urgency/emergency basis in four patients $(4 / 6,67 \%)$ (Figures 3-5). Additional pre-operative balloon atrial septectomy was necessary in one child. None underwent cardiac transplantation. Mean age at surgery was $1.1 \pm 0.4$ years ( 1 day -5.5 years). Basic principles of surgery consisted in radical tumor excision without causing damage to other cardiac structures. All operations were performed through median sternotomy utilizing cardiopulmonary bypass with aortic and bicaval cannulation. Complete excision of the mass was achievable in 5 cases. In one case, only explorative sternotomy and tumor biopsy was done due to impossibility to respect the mass, which infiltrated diffusely both ventricles (Figures 1-3). Reoperation was necessary in 2 cases $(2 / 5,40 \%)$ for recurrence of PCT (mean time at recurrence 3.4 years, range 2.5-4.3 years). Correction of associated CHDs was necessary in one patients and it consisted in closure of an atrial (ASD) and ventricular (VSD) septal defect. In 2 patients, tricuspid valvuloplasty was necessary after tumor excision due to the location on tumor itself.

Surgical mortality occurred in one patient who required postoperative support with veno-arterial extracorporeal membrane oxygenation (V-A ECMO) and iNO, because of refractory hypoxia. Six days after weaning from ECMO, he died because of sepsis and recurrent respiratory failure. Overall mortality $(2 / 6,22 \%)$ was similar between operated and non-operated group of patients with one case observed in each group, since we considered as "nonoperated" the newborn presenting with a diffusely infiltrating and non resectable tumor, who died because of severe biventricular dysfunction and obstruction (Figure 3). Histological examination of the mass was performed in all surgically managed patients. It revealed 2 cases of familial fetal type of rhabdomyoma, 1 rhabdomyoma, 2 myxoma and 1 calcified amorphous tumor (CAT) of the heart (Figure 4), (Table 2).

\section{F/U Assessment}

Two patients were lost at F/U (2/14, 14\%). One case decided for termination of pregnancy $(1 / 14,77 \%)$. Regarding the remaining 11 cases, median follow-up time was 3.1 years ( 24 days - 10 years). Overall survival was $82 \%(9 / 11 ; 4$ in the surgical-managed group, 5 in the medically managed group) with only two in-hospital deaths. Seven patients underwent conservative approach due to clinical and hemodynamic stability. In this group of patients, one case faced with complete spontaneous regression of the cardiac mass $(1 / 7,14 \%)$ and 3 patients faced with spontaneous reduction of the dimension of the mass $(3 / 7,43 \%)$. Two patients needed treatment with Everolimus $(2 / 7,29 \%)$ to induce rapid mass reduction, avoid the risk of inflow and outflow obstruction and allowed patients to overcome neonatal critical phase without complications.

One Everolimus-treated patient underwent efficacious B-blocker antiarrhythmic therapy because of supraventricular tachycardia with hemodynamic impairment. In medical managed group of patients, the exact type of PCT was impossible to define due to lack of histological confirmation by biopsy. Nevertheless, based on the clinical course and echocardiographic characteristics, masses were judged as rhabdomyomas. At last examination, none of the patients had evidence of recurrence. All surviving patients were in NYHA functional class I. Physical examination, arterial blood pressure, heart rate, blood oxygen saturation and ecg were normal in all but one case (complete right bundle branch block).

\section{Discussion}

Benign PCTs are rare diseases [1,2] with extremely different clinical course ranging from spontaneous regression, especially for rhabdomyomas of small dimension in younger patients $[1,3,4]$ to progressive and severe hemodynamic impairment due to cardiac inflow and outflow obstruction, arrhythmias, valve dysfunction and embolization [1-4]. Echography is the cornerstone for prenatal 
diagnosis of cardiac tumors. The first in utero diagnosis of PCT dates 1982 [5]. Since then, improvement in diagnostic techniques made fetal diagnosis of cardiac masses generally feasible. Up to date, fetal echocardiography is still the gold standard for prenatal identification of PCTs, as fetal CT or MRI do not seem to improve significantly the ability to better define disease and type of tumors [5]. Major referral indications for fetal echocardiography, in the present study as well as in prior experience [5], are: suspicion of CHD and of tuberous sclerosis, family history of CHD; high risk of fetal chromosome abnormality. Complete fetal echocardiography should be performed if a PCT is suspected [6].

Number, dimension, site and echographic characteristics of PCT should be collected. Possible associated CHDs should be carefully researched. Signs of possible fetal heart failure (ventricular dysfunction, fetal bradi or tachyarrhythmia, insufficiency of atrioventricular valves, outflow tract obstruction, pericardial effusion, ascites, fetal hydrops, and growth retardation) should be carefully investigated and, if present, carefully monitored during pregnancy, as some PCTs can progress in utero up to fetal demise. Obstetrical evaluation is essential to define possible non-cardiac associated malformations and chromosome abnormality and to follow fetal growth and fetal-maternal wellness, as some cases may need trans-placental therapy or early delivery. On the other hand, caution on invasive strategies (termination of pregnancy, early delivery, trans-placental therapy, drainage of fetal pericardial effusion) should be performed as some cardiac fetal masses regress spontaneously. Prognosis of cardiac masses was not influenced by prenatal diagnosis in the present study, although prenatal diagnosis of PCT remains extremely important to define the management strategy.

It allows not only diagnosis of PCT but also of associated CHD, of extra-cardiac malformations or chromosome abnormalities that may play a role in the global prognosis of the disease, in the follow up of the fetal/maternal wellness and eventually the termination of pregnancy. Moreover, it allows planning of the delivery in a tertiary center, where intensive neonatal care, cardiac intensive care and cardiac surgical options are promptly available, if required. Prenatal diagnosis is important for both parents, who may be aware of the possible postnatal worsening, and medical team, who may build the best strategy to offer patients and families appropriate therapeutic opportunities. Regardless of clinical behavior and the need of intervention, prenatal diagnosis is also essential to describe the natural history of PCT as some of them may regress partially or even completely during fetal and postnatal life. Even in the postnatal period, 2-D and Doppler echocardiography represent the first line, and often the only one needed, diagnostic technique allowing the definition of the presence of an intra-cardiac mass, number, dimension, location and hemodynamic implication.

Cardiac CT scan and cMRI are second line postnatal techniques useful to achieve a more precise definition of location of the mass, relationship with the surrounding structures and tissue characterization of the tumor that corroborates with pathological study for defining the histological nature of it [7-10]. In our series, cardiac CT was performed only in 2 cases in order to better define the relationship between the mass, the coronary arteries and the surrounding structures. Moreover, cMRI was necessary in only 2 older patients (mean age at postnatal diagnosis 1,5 years). At younger age, cMRI is technically challenging due to high cardiac frequencies, possible patient's movements and needing of pharmacological sedation for quite long time. Clinical presentation of PCT is extremely variable and so does optimal management strategy. In our experience, symptoms are present in less than a half of cases and do not correlate with histological type of the tumor and age at diagnosis but with the location of the tumor, as previously described [12-14]. As compared to asymptomatic pts, symptomatic pts need more frequently pharmacological therapy and cardiocirculatory/respiratory support and have a higher risk of mortality.

In the present series, mortality occurred in two patients $(2 / 13,15 \%)$, who were both symptomatic at the time of diagnosis and needed intensive care support. It is evident that mortality and symptoms can be related to a more severe form of presentation of the disease which causes hemodynamic impairment. Those findings correlate well with other reports [1,2,14]. Surgical treatment is usually indicated in case of symptoms or hemodynamic instability and in asymptomatic patients in whom instrumental data indicate a significant risk of life threatening conditions, such as risk of embolization, inflow and outflow tracts obstruction, valve dysfunction and arrhythmias. These complications usually are associated with certain types of PCT such as cardiac fibroma (conduction defects and obstruction) and cardiac myxoma (valve obstruction and embolization from thrombosis or tumor fragment) $[1,11]$. In these cases, even if regression of cardiac tumors might have occurred, surgical treatment is considered a prophylactic strategy to prevent possible fatal complications $[4,11,13]$. In our cohort of patients, indication for surgical treatment were cardiac obstruction leading to hemodynamic instability and risk of tumor embolization.

Primary goal of surgical treatment is complete excision of the mass $[15,16]$. In case of myocardium infiltration and closeness with coronary arteries or other noble heart structures, where complete resection of the tumor is not feasible, partial resection of PCT is preferable and safe $[4,13,17]$. Surgical strategy for the treatment of PCT, even in early childhood, carries an acceptable mortality risk $[4,13,14]$. In our limited and heterogeneous experience, complete surgical resection of the mass was feasible in the majority of pts, even early in infancy. Mortality occurred only in two patients $(2 / 13,15 \%)$ because of severe, refractory pulmonary hypertension due to tumor fragmentation and pulmonary embolism in one case and because of severe biventricular obstruction not amenable to surgical excision in the other case. In the present series surgery was necessary in six patients $(6 / 13,46 \%)$, of whom 4 survived $(67 \%)$. The rate of re-operation for disease occurrence was $40 \%(2 / 5)$ with a mean time to recurrence of 3.4 years. Recurrence occurred in a patient who underwent a myxoma resection and the other case had a fetal type rhabdomyoma.

This high rate of occurrence is in line with the complexity of the disease and the behavior of the tumor. Our histological findings match known epidemiological data [2,18] as rhabdomyoma is the most frequent benign tumor diagnosed within the first month of life, followed by cardiac mixoma. Drug therapy can be needed at 
time of diagnosis to support the cardio-circulatory system as bridge to recovery (PGE) or bridge to surgery (PGE, inotropic support, diuretic drugs). It can be needed at long term to treat or prevent arrhythmias. More recently, Everolimus therapy seems to speed up the regression of cardiac rhabdomyomas, even if no international consensus exists regarding indication for its use [19-21]. We decided to start this therapy in 2 neonatal pts in order to try not to perform surgical excision of voluminous cardiac rhamdomyomas. Long survivals remain asymptomatic, in good functional class and with normal physical and somatic development [22].

\section{Conclusion}

In conclusion, prenatal diagnosis is crucial to plan delivery and neonatal management in a tertiary care Centre, as some benign PCTs require neonatal intensive care management. Complete excision of the mass usually is feasible and safe. Surgical mortality is generally low. Prognosis is good as patients are generally free from recurrence and symptoms after surgical treatment. Team work is essential to correctly manage neonates and children affected by PCT.

\section{Limitations of the Study}

Limitations of the present study are inherent with the retrospective nature, small number of patients and different baseline demography of the population. Also, the type of disease itself represents an important limitation considering the extremely rare incidence of PCT. Nonetheless, the present is amongst the largest single Institution clinical series of primary cardiac tumors in infants and children.

\section{References}

1. Freedom RM, Lee KJ, Mac Donald C, Taylor G (2000) Selected aspects of cardiac tumors in infancy and childhood. Pediatr Cardiol 21(4): 299316.

2. Keane J, Fyler D, Lock J (2006) Nadas' Pediatric Cardiology. $2^{\text {nd }}$ (Edn.)., Saunders.

3. Beghetti M, Gow RM, Haney I, Mawson J, Williams WG, et al. (1997) Pediatric primary benign cardiac tumors: a 15-year review. Am Heart J 134(6): 1107-1114.

4. Günther T, Schreiber C, Noebauer C, Eicken A, Lange R (2008) Treatment strategies for pediatric patients with primary cardiac and pericardial tumors: a 30-year review. Pediatr Cardiol 29(6): 1071-1076.

5. Yagel S, Silverman NH, Gembruch U, Cohen SM (2008) Fetal Cardiology. $2^{\text {nd }}($ Edn.)., Informa Healthcare.

6. Mehta SM, Myers JL (2000) Congenital heart surgery nomenclature and database project: cardiac tumors. Ann Thorac Surg 69(4): S358-368.

7. Beroukhim RS, Prakash A, Buechel ER, Cava JR, Dorfman AL, et al. (2011) Characterization of cardiac tumors in children by cardiovascular magnetic resonance imaging: a multicenter experience. J Am Coll Cardiol 58(10): 1044-1054.
8. Araoz PA, Mulvagh SL, Tazelaar HD, Julsrud PR, Breen JF (2000) CT and MR imaging of benign primary cardiac neoplasms with echocardiographic correlation. Radiographics 20(5): 1303-1319.

9. O’Donnell DH, Abbara S, Chaithiraphan V, Yared K, Killeen RP, et al. (2009) Cardiac tumors: optimal cardiac MR sequences and spectrum of imaging appearances. AJR 193(2): 377-387.

10. Gulati G, Sharma S, Kothari SS, Juneja R, Saxena A, et al. (2004) Comparison of Echo and MRI in the imaging evaluation of intracardiac masses. Cardiovasc Intervent Radiol 27(5): 459-469.

11. Cho JM, Danielson GK, Puga FJ, Dearani JA, McGregor CG, et al. (2003) Surgical resection of ventricular cardiac fibromas: early and late results. Ann Thorac Surg 76(6): 1929-1934.

12. Viscardi F, Errico G, Schiavo N, Biban P, Mazzucco A, et al. (2009) Familial fetal-type rhabdomyoma of the tricuspid valve in the neonate: Malignant course for a benign disease. J Thorac Cardiovasc Surg 137(3): 751-753.

13. Padalino MA, Vida VL, Boccuzzo G, Tonello M, Sarris GE, et al. (2012) Surgery for primary Cardiac Tumors in children early and late results in a Multicenter European Congenital Heart Surgeons Association Study. Circ 126(1): 22-30.

14. Padalino MA, Basso C, Milanesi O, Vida VL, Svaluto Moreolo G, et al. (2005) Surgically treated primary cardiac tumors in early infancy and childhood. J Thorac Cardiovasc Surg 129(6): 1358-1363.

15. Schmaltz AA, Apitz J (1981) Primary heart tumors in infancy and childhood. Report of four cases and review of literature. Cardiology 67(1): 12-22.

16. Takach TJ, Reul GJ, Oft DA, Cooley DA (1996) Primary Cardiac Tumors in Infants and Children: Immediate and Long-Term Operative Results. Ann Thorac Surg 62(2): 559-564.

17. Ceithaml EL, Midgley FM, Perry LW, Dullum M (1990) Intramural ventricular fibroma in infancy: survival after partial excision. Ann Thorac Surg 50(3): 471-472.

18. Allen HD, Driscoll DJ, Shaddy RE, Feltes TF (2013) Moss \& Adams' Heart Disease in Infants, Children, and Adolescents: Including the Fetus and Young Adult. $8^{\text {th }}$ (Edn.)., LWW.

19. Hinton RB, Prakash A, Romp RL, Krueger DA, Knilans TK (2014) International Tuberous Sclerosis Consensus Group. Cardiovascular manifestations of tuberous sclerosis complex and summary of the revised diagnostic criteria and surveillance and management recommendations from the International Tuberous Sclerosis Consensus Group. J Am Heart Assoc 3(6): e001493.

20. Moavero R, Romagnoli G, Graziola F, Curatolo P (2015) Mammalian Target of Rapamycin Inhibitors and Life-Threatening Conditions in Tuberous Sclerosis Complex. Semin Pediatr Neurol 22(4): 282-294.

21. MacKeigan JP, Krueger DA (2015) Differentiating the mTOR inhibitors everolimus and sirolimus in the treatment of tuberous sclerosis complex. Neuro Oncol 17(12): 1550-1559.

22. Aw F, Goyer I, Raboisson MJ, Boutin C, Major P, et al. (2017) Accelerated Cardiac Rhabdomyoma Regression with Everolimus in Infants with Tuberous Sclerosis Complex. Pediatr Cardiol 38(2): 394-400. 


\section{ISSN: 2574-1241}

DOI: 10.26717/BJSTR.2018.07.001483

Giovanni Battista Luciani. Biomed J Sci \& Tech Res

(C)

Submission Link: https://biomedres.us/submit-manuscript.php

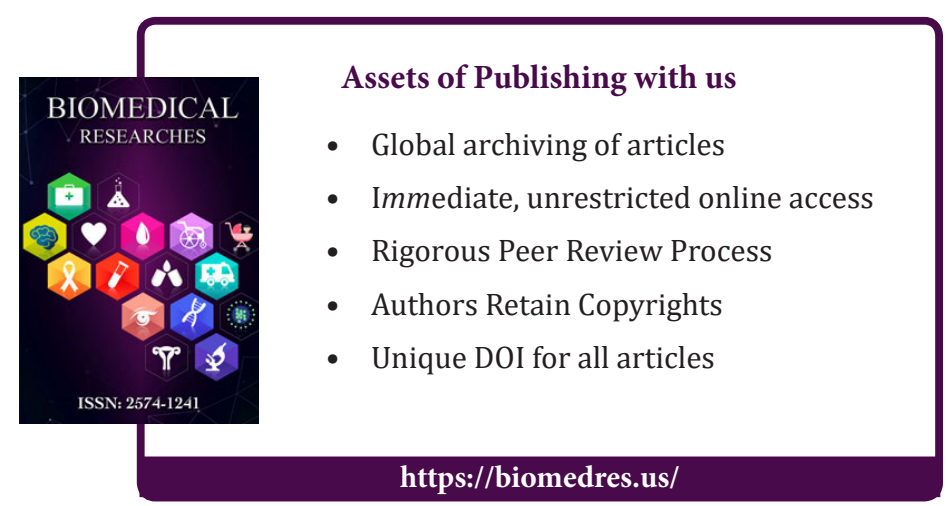

